\title{
Recent Results on Neutrino Masses
}

\author{
José W. F. Valle \\ Instituto de Física Corpuscular - C.S.I.C. \\ Departament de Física Teòrica, Universitat de València \\ 46100 Burjassot, València, Spain \\ http://neutrinos.uv.es
}

\begin{abstract}
I review the main options one has of introducing mass to neutrinos, including broken $\mathrm{R}$-parity models, as well as the constraints on neutrino properties that follow from astrophysics, cosmology as well as laboratory observations.
\end{abstract}

\section{Introduction}

One of the most unpleasant features of the Standard Model (SM) is that the masslessness of neutrinos is not dictated by an underlying principle, such as gauge invariance, in the case of the photon: the SM simply postulates that neutrinos are massless and, as a result, most of their properties are trivial. Massless neutrinos would be exceptional particles, since no other such fermions exist. If massive, neutrinos would present another puzzle, of why are their masses so much smaller than those of the charged fermions. The fact that neutrinos are the only electrically neutral elementary fermions means that they could be Majorana-type, the most fundamental one. This may hold the key to why neutrino masses are small: they break lepton number symmetry. This feature is encountered in many extensions of the SM. However, at the present stage of affairs, theory alone cannot make definite predictions for the magnitude of particle masses, and neutrinos are no exception. We simply do not know the scale responsible for neutrino mass, nor the underlying mechanism. Last but not least, one lacks a theory for the Yukawa couplings. In what follows I will discuss the main options one has of introducing mass to neutrinos, and over-view the present information that follows from astrophysics, cosmology as well as laboratory observations.

\section{Models of Neutrino Mass}

One of the most popular approaches to generate neutrino masses is Unification. Indeed, in trying to understand the origin of parity violation in the weak interaction by ascribing it to a spontaneous breaking phenomenon, in the same way as the $\mathrm{W}$ and $\mathrm{Z}$ acquire their masses in the SM, one arrives at the so-called left-right symmetric extensions $S U(2)_{L} \otimes S U(2)_{R} \otimes U(1)$ [1], $S U(4) \otimes S U(2) \otimes S U(2)$ [2] or $S O(10)$ [3], in some of which the masses of the light neutrinos are obtained by diagonalizing the following mass matrix in the basis $\nu, \nu^{c}$

$$
\left[\begin{array}{cc}
0 & D \\
D^{T} & M_{R}
\end{array}\right]
$$


where $D$ is the standard $S U(2) \otimes U(1)$ breaking Dirac mass term and $M_{R}=M_{R}^{T}$ is the isosinglet Majorana mass associated to the violation of the extended gauge symmetry. In the seesaw approximation, one finds

$$
M_{e f f}=-D M_{R}^{-1} D^{T}
$$

In general, however, one also has a $\nu \nu$ term whose size is expected to be also suppressed by the righthanded breaking scale. This way one can naturally explain the smallness of neutrino masses. Even though $M_{R}$ should be large, its magnitude heavily depends on the model. As a result one can not make any solid prediction for the light neutrino mass spectrum generated through the seesaw mechanism, nor for the resulting pattern of mixing 郻. In fact this freedom has been exploited in model building in order to account for an almost degenerate neutrino mass spectrum presently suggested by solar and atmospheric neutrino data as well as the recent observations on structure formation [5].

Although very attractive, Unification is by no means the only way to generate neutrino masses. There is a large diversity of other possible schemes which do not require any new large mass scale. For example, it is possible to start from an extension of the lepton sector of the $S U(2) \otimes U(1)$ theory by adding a set of two 2-component isosinglet neutral fermions, denoted $\nu^{c}{ }_{i}$ and $S_{i}$, to each generation. In this case there is an exactly conserved L symmetry that keeps neutrinos strictly massless, as in the $\mathrm{SM}$, leading to the following form for the neutral mass matrix (in the basis $\nu, \nu^{c}, S$ )

$$
\left[\begin{array}{ccc}
0 & D & 0 \\
D^{T} & 0 & M \\
0 & M^{T} & 0
\end{array}\right]
$$

This form has also been suggested in various theoretical models [6], including many of the superstring inspired models [7]. In the latter case the zeros of eq. (3) arise due to the absence of Higgs fields to provide the usual Majorana mass terms. Although neutrinos are massless in this model, they are mixed in the leptonic charged current in a non-trivial way which cannot be eliminated by field redefinitions. Conventional neutrino oscillation searches in vacuo are insensitive to this mixing. However, such neutrinos may resonantly convert in the dense medium of a supernova [8, 9]. One can also introduce non-zero masses in this model through a $\mu S S$ term that could be proportional to the vacuum expectation value of a singlet field $\sigma[10]$. In contrast to the seesaw scheme, neutrino masses would be directly proportional to $\langle\sigma\rangle, m_{\nu} \propto\langle\sigma\rangle$. The massless neutrino model characterized by eq. (3) provides a conceptually simple and phenomenologically rich extension of the Standard Model, which brings in the possibility that a wide range of new phenomena be sizeable. These have to do with neutrino mixing, universality, flavour and $\mathrm{CP}$ violation in the lepton sector [11, 12], as well as direct effects associated with neutral heavy lepton production at high energy colliders [13]. The rates for loop-induced lepton flavour and CP nonconservation effects in this model are precisely calculable [11, 12, 14] and can be large as they are not restricted by neutrino masses, only by weak universality violation constraints, which are weaker. The only other example I know of getting sizeable lepton flavour violation (LFV) without neutrino masses is the supersymmetric mechanism first described in ref. [15]. It is interesting to realize that LFV does 


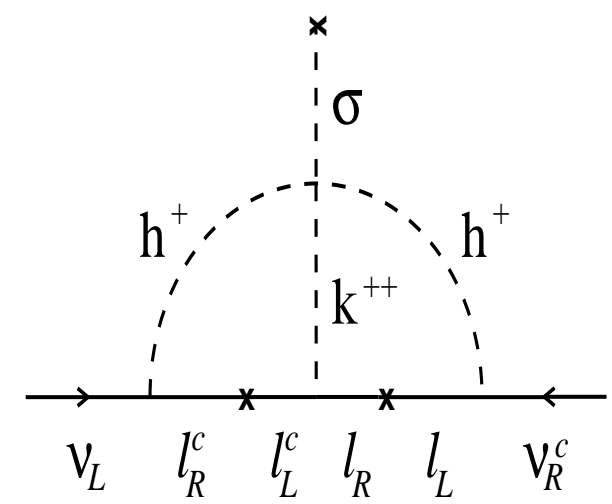

Figure 1: Two-loop-induced Neutrino Mass.

not require neutrino masses, unlike total-L-violating processes, such as neutrino-less double beta decay, see below.

There is also a large variety of radiative schemes to generate neutrino masses. The prototype models of this type are the Zee model and the model suggested by Babu 16. In these models lepton number is explicitly broken, but it is easy to enforce the spontaneous breaking of lepton number by adding a single scalar boson $\sigma$, as in the version of ref. [17]. Note that the neutrino mass arising from the diagram shown in Fig. (1) goes to zero as $\langle\sigma\rangle \rightarrow 0$.

The seesaw and the radiative mechanisms of neutrino mass generation may be combined. Supersymmetry with broken R-parity provides a very elegant mechanism to do this, and can explain the origin of neutrino mass, as well as mixings [18]. Here I focus on the simplest unified supergravity version of the model with bilinear breaking of R-parity, characterized universal boundary conditions for the soft breaking parameters [18]. In this model the tau neutrino (which is the fifth in the following matrix) acquires a mass, due to the mixing between the neutrino and the neutralinos given in the matrix [19]

$$
\left[\begin{array}{ccccc}
M_{1} & 0 & -\frac{1}{2} g^{\prime} v_{1} & \frac{1}{2} g^{\prime} v_{2} & -\frac{1}{2} g^{\prime} v_{3} \\
0 & M_{2} & \frac{1}{2} g v_{1} & -\frac{1}{2} g v_{2} & \frac{1}{2} g v_{3} \\
-\frac{1}{2} g^{\prime} v_{1} & \frac{1}{2} g v_{1} & 0 & -\mu & 0 \\
\frac{1}{2} g^{\prime} v_{2} & -\frac{1}{2} g v_{2} & -\mu & 0 & \epsilon_{3} \\
-\frac{1}{2} g^{\prime} v_{3} & \frac{1}{2} g v_{3} & 0 & \epsilon_{3} & 0
\end{array}\right]
$$

This matrix is a truncation of the more complete one that one finds in dynamical models with spontaneous breaking of $\mathrm{R}$-parity and which necessarily contain singlet neutrino superfields [20]. Here I focus on the simplest truncated model with explicit violation of R-parity. Contrary to a popular misconception, the bilinear violation of $\mathrm{R}$-parity implied by the parameter $\epsilon_{3}$ is physical, and can not be rotated away. One can show that by going to a basis in which $\epsilon_{3}$ is eliminated from the superpotential one gets exactly the same results as in the original un-rotated basis [21]. A minimal supergravity version of this model contains only one extra free parameter in addition to those of the minimal $\mathrm{R}$-parity conserving supergravity model, as the $\epsilon_{3}$ and the $v_{3}$ are related by a minimization condition [18]. The assumed universality of the soft breaking parameters implies that the value of $\epsilon_{3}$ is induced radiatively, due 


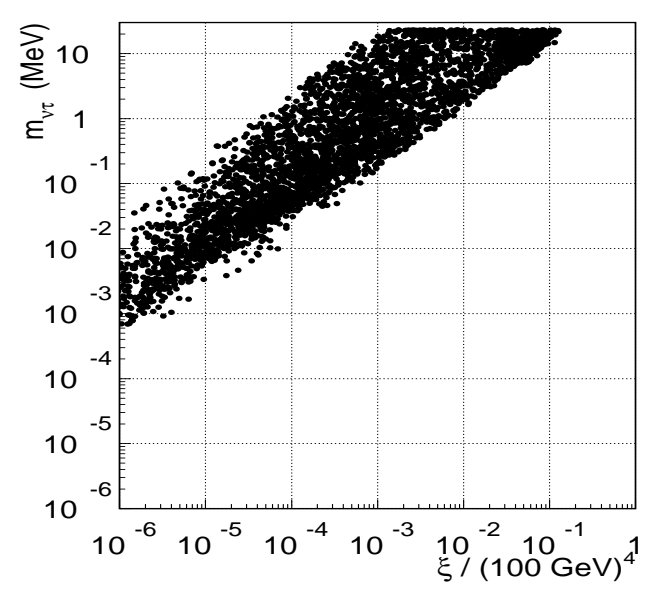

Figure 2: Tau neutrino mass versus $\epsilon_{3}$

to the effect of the non-zero bottom quark Yukawa coupling $h_{b}$ in running the renormalization group equations from the unification scale down to the weak scale [18]. This makes $\epsilon_{3}$ calculable. Thus eq. (4) is analogous to a see-saw type matrix eq. (1), in which the sector analogous to $M_{R}$ (neutralinos) lies at the weak scale, while the rôle of the Dirac entry $D$ is played by the $\epsilon_{3}$, which is, in a sense, a radiatively induced quantity. From this point of view, the mechanism is a hybrid see-saw-like scheme, with the Majorana $\nu_{\tau}$ mass induced by the mixing of neutrinos with Higgsinos or gauginos. The $\nu_{\tau}$ mass induced this way depends quadratically on an effective parameter $\xi$ defined as $\xi \equiv\left(\epsilon_{3} v_{1}+\mu v_{3}\right)^{2}$ and characterizing the violation of $\mathrm{R}-$ parity either through gaugino $\left(v_{3}\right)$ or Higgsino mixing $\left(\epsilon_{3}\right)$. In Fig. (2) we display the allowed values of $m_{\nu_{\tau}}$, which can be quite low even for sizeable values of $\epsilon_{3}$, due to the possible cancellation between the two terms in $\xi$. In the unified supergravity version of the model with universal soft masses this cancellation happens automatically. The value of $\xi$ becomes calculable in terms of $h_{b}$, leading to a naturally suppressed $m_{\nu_{\tau}}$ in the $\mathrm{KeV}$ range. Notice that $\nu_{e}$ and $\nu_{\mu}$ remain massless in this approximation, even if the other two parameters $\epsilon_{1}$ and $\epsilon_{2}$ are added to complete the model for the first two families. This is an old result related to the projective nature of the neutrino mass matrix in these models [22]. The $\nu_{\mu}$ will get mass either from scalar loop contributions in Fig. (3) [23], or by mixing with singlets in models with spontaneous breaking of R-parity [20]. This leads to a solution to the solar neutrino puzzle via $\nu_{e}$ to $\nu_{\mu}$ conversions. In short, broken $\mathrm{R}$-parity supersymmetry provides an elegant mechanism for generating neutrino masses and mixings. It is important to notice that even when the neutrino masses are small, the corresponding R-parity violating effects can be large. For example the lightest neutralino typically happens inside the detector, giving rise to remarkable signatures at accelerator experiments such as LEP/LHC.

Other than the seesaw scheme, none of the above models requires a large mass scale. In all of them one can implement the spontaneous violation of the global lepton number symmetry leading to neutrino masses that scale directly proportional to the lepton-number scale $\langle\sigma\rangle$ or some positive power of it. Such low-scale Majoron models are very attractive and lead to a rich phenomenology, as the 


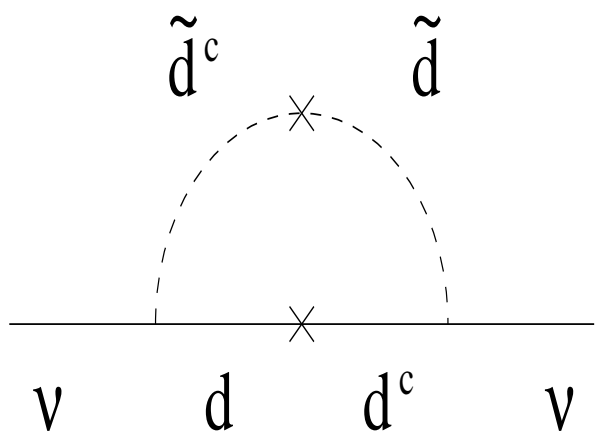

Figure 3: Scalar loop contributions to neutrino masses

extra particles required have masses at scales that could be accessible to present experiments. One remarkable example is the possibility invisibly decaying Higgs bosons [24].

The above discussion illustrates the enormous wealth of phenomenological possibilities in the neutrino sector. These reach well beyond the realm of conventional neutrino experiments, including also signatures that can be probed, though indirectly, at high energy accelerators. Unfortunately, as already mentioned, neutrino masses are not predicted by theory and it is up to observation to search for any possible clue. Given the theoretical uncertainties in predicting neutrino masses from first principles, one must turn to observation. Here the information comes from laboratory, cosmology and astrophysics.

\section{Laboratory Limits}

The only model-independent laboratory limits on neutrino mass follow purely from kinematics [25]:

$$
m_{\nu_{e}} \lesssim 5 \mathrm{eV}, \quad m_{\nu_{\mu}} \lesssim 170 \mathrm{keV}, \quad m_{\nu_{\tau}} \lesssim 18 \mathrm{MeV}
$$

The limit on the $\nu_{e}$ mass comes from tritium beta decay [26] while further results from the Mainz experiment are eagerly awaited. The $\nu_{\mu}$ mass limit comes from PSI (90\% C.L.) [27], with further improvement limited by the uncertainty in the $\pi^{-}$mass. On the other hand, the best $\nu_{\tau}$ mass limit now comes from high energy LEP experiments [28] and may be substantially improved at a future tau-charm factory [29].

The most stringent neutrino oscillation bounds come from reactor experiments [30] $\left(\bar{\nu}_{e}-\nu_{x}\right.$ oscillations); from meson factory oscillation experiments (KARMEN [31], LSND [32]) and from high-energy accelerator experiments E531 and E776 [33] $\left(\nu_{\mu}-\nu_{\tau}\right)$. A recent search for $\nu_{\mu}$ to $\nu_{e}$ oscillations by the LSND collaboration using $\nu_{\mu}$ from $\pi^{+}$decay in flight 34] is consistent with their previously reported $\bar{\nu}_{\mu}$ to $\bar{\nu}_{e}$ oscillation evidence. However, a recent result from NOMAD rules out part of the LSND region. The future lies in searches for oscillations using reactor or accelerator neutrino beams directed to farout underground detectors, with good prospects for Chooz and Palo Verde, as well as the long-baseline experiments proposed at KEK, CERN and Fermilab. 


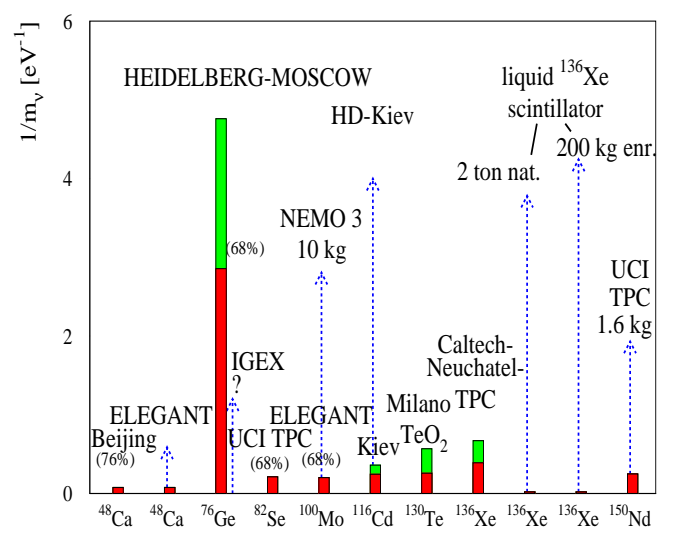

Figure 4: Sensitivity of $\beta \beta_{0 \nu}$ experiments.

If neutrinos are of Majorana type a new form of nuclear double beta decay would take place in which no neutrinos are emitted in the final state, i.e. the process by which an $(A, Z-2)$ nucleus decays to $(A, Z)+2 e^{-}$. Such process would arise from a virtual exchange of Majorana neutrinos. Unlike ordinary double beta decay, the neutrino-less process violates total lepton number L and its existence would indicate the Majorana nature of neutrinos. Because of the phase space advantage, this process is a very sensitive probe of the nature of neutrinos. Present limits as well as future prospects are illustrated in Fig. (4), taken from ref. [35]. Note that this bound depends to some extent on the relevant nuclear matrix elements characterising this process [36] and that the effective particle physics parameter $\langle m\rangle$ involves both neutrino masses and mixings. Thus, although rather stringent, this limit may allow very large individual neutrino masses, as there may be strong cancellations between different neutrino types. These could follow from suitable symmetries. For example, the decay vanishes if the intermediate neutrinos are Dirac-type, as a result of the corresponding lepton number symmetry [37].

Neutrino-less double beta decay has a great conceptual importance. It has been first shown in ref. 38] that in a gauge theory of the weak interactions a non-vanishing $\beta \beta_{0 \nu}$ decay rate requires neutrinos to be Majorana particles, irrespective of which mechanism induces it. A recent application of this theorem to supersymmetry was discussed here by Hirsch [39].

\section{The Cosmological Density Limit}

The oldest cosmological bound on neutrino masses follows from avoiding the overabundance of relic neutrinos 40

$$
\sum m_{\nu_{i}} \lesssim 92 \Omega_{\nu} h^{2} e V
$$

where $\Omega_{\nu} h^{2} \leq 1$ and the sum runs over all species of isodoublet neutrinos with mass less than $O(1 \mathrm{MeV})$. Here $\Omega_{\nu}=\rho_{\nu} / \rho_{c}$, where $\rho_{\nu}$ is the neutrino contribution to the total density and $\rho_{c}$ is the critical density. The factor $h^{2}$ measures the uncertainty in the present value of the Hubble parameter, $0.4 \leq h \leq 1$, 
and $\Omega_{\nu} h^{2}$ is smaller than 1 . For the $\nu_{\mu}$ and $\nu_{\tau}$ this bound is much more stringent than the laboratory limits eq. (5).

Apart from the experimental interest, an $\mathrm{MeV}$ tau neutrino is interesting from the point of view of structure formation [41]. Such $\nu_{\tau}$ masses are theoretically viable as the constraint in eq. (6) holds only if neutrinos are stable on cosmological time scales. In models with spontaneous violation of total lepton number [42] neutrinos can decay into a lighter neutrino plus a majoron, for example [43],

$$
\nu_{\tau} \rightarrow \nu_{\mu}+J
$$

or have sizeable annihilations to these majorons,

$$
\nu_{\tau}+\nu_{\tau} \rightarrow J+J
$$

The possible existence of fast decay and/or annihilation channels could eliminate relic neutrinos and therefore allow them to have higher masses, as long as the lifetime is short enough to allow for an adequate red-shift of the heavy neutrino decay products. These 2-body decays can be much faster than the visible modes, such as radiative decays of the type $\nu^{\prime} \rightarrow \nu+\gamma$, which would also be highly constrained by astrophysics and cosmology (for a detailed discussion see ref. [40]).

A general method to determine the Majoron emission decay rates of neutrinos was first given in ref. [44]. The resulting decay rates are rather model-dependent and will not be discussed here. Explicit neutrino decay lifetime estimates are given in ref. [20, 43, 45]. The conclusion is that there are many ways to make neutrinos sufficiently short-lived and that all mass values consistent with laboratory experiments are cosmologically acceptable.

\section{The Cosmological Nucleosynthesis Limit}

Stronger limits on neutrino lifetimes or annihilation cross sections arise from cosmological nucleosynthesis. Recent data on the primordial deuterium abundance [46] have stimulated a lot of work on the subject [47]. If a massive $\nu_{\tau}$ is stable on the nucleosynthesis time scale, $\left(\nu_{\tau}\right.$ lifetime longer than $\sim 100$ sec), it can lead to an excessive amount of primordial helium due to its large contribution to the total energy density. This bound can be expressed through an effective number of massless neutrino species $\left(N_{\nu}\right)$. If $N_{\nu}<3.4-3.6$, one can rule out $\nu_{\tau}$ masses above $0.5 \mathrm{MeV}$ 48. If we take $N_{\nu}<4$ the $m_{\nu_{\tau}}$ limit loosens accordingly. However it has recently been argued that non-equilibrium effects from the light neutrinos arising from the annihilations of the heavy $\nu_{\tau}$ 's make the constraint a bit stronger in the large $m_{\nu_{\tau}}$ region [49], ruling out all $\nu_{\tau}$ masses oi this range. One can show, however that in the presence of the $\nu_{\tau}$ annihilations in eq. (8) the nucleosynthesis $m_{\nu_{\tau}}$ bound is substantially weakened or eliminated [50]. Fig. 5 gives the effective number of massless neutrinos equivalent to the contribution of a massive $\nu_{\tau}$ for different values of the coupling $g$ between $\nu_{\tau}$ 's and $J$ 's, expressed in units of $10^{-5}$. For comparison, the dashed line corresponds to the SM $g=0$ case. One sees that for a fixed $N_{\nu}^{\max }$, a wide 


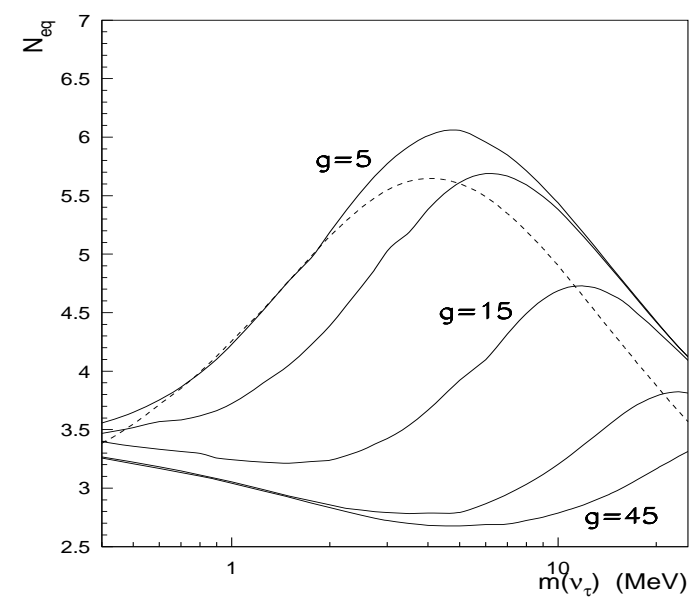

Figure 5: A few $\mathrm{MeV} \nu_{\tau}$ annihilating to majorons can lower the primordial helium abundance.

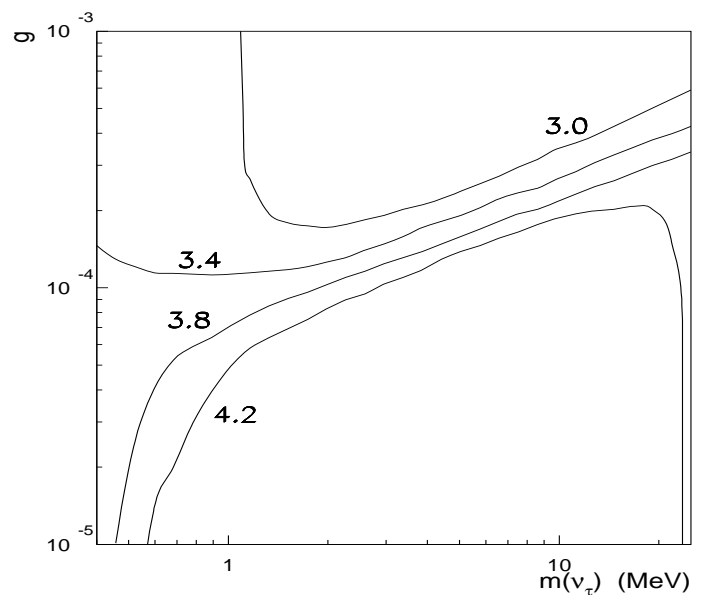

Figure 6: The region above each curve is allowed for the corresponding $N_{e q}^{\max }$.

range of tau neutrino masses is allowed for large enough values of $g$. As long as $g$ exceeds a few times $10^{-4}$ no $\nu_{\tau}$ masses below the LEP limit can be ruled out. One can express the above results in the $m_{\nu_{\tau}}-g$ plane, as shown in figure 6. One sees that the $\nu_{\tau}$ mass constraints from primordial nucleosynthesis can be substantially relaxed, for values of $g\left(m_{\nu_{\tau}}\right)$ which are reasonable in many majoron models such as those in ref. [24, 43, 50, 51]. Similar depletion in massive $\nu_{\tau}$ relic abundance also happens if the $\nu_{\tau}$ is unstable on the nucleosynthesis time scale as will happen in many Majoron models [52].

\section{Supernova Limits}

There are a variety of limits on neutrino parameters that follow from astrophysics [53], including the SN1987A observations, as well as considerations from supernova (SN) theory, such as SN dynamics [54] and SN nucleosynthesis [55]. Here I briefly discuss some recent examples of how supernova physics constrains neutrino parameters in various neutrino conversion scenarios.

The observation of the energy spectrum of the SN1987A $\bar{\nu}_{e}$ 's may be used to provide very stringent 
Fig. 2

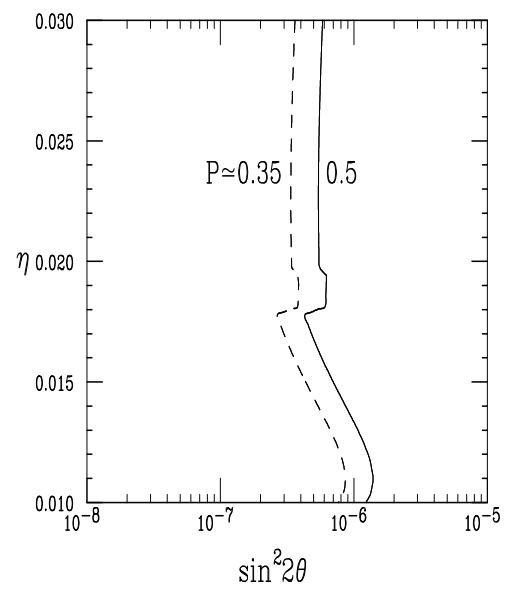

Figure 7: SN1987A bounds on massless neutrino mixing.

constraints on the parameters characterizing resonant neutrino conversions that could take place in the dense supernova medium [56]. This can be applied to various neutrino conversion scenarios in order to constrain the relevant parameters. As an example, the regions to the right of the curves in Fig. (7), taken from ref. [9], are ruled out by SN1987A data, while those to the left are allowed. This is very surprising indeed, as there is no way to constrain the mixing of massless neutrinos in the laboratory, since no vacuum neutrino oscillations are possible. As another illustration consider the case of resonant neutrino conversions (massless or massive) induced by flavour changing neutral current (FCNC) neutrino interactions of broken R-parity models. The restrictions that follow from the observed $\bar{\nu}_{e}$ energy spectra from SN1987A are shown in Fig. (8), taken from ref. [57], for the case where neutrino masses are in the hot dark matter range.

It has been noted that neutrino conversions may play an important rôle in SN $r$-process nucleosynthesis [55]. This has been used in order to obtain information on various neutrino conversion scenarios. In Fig. (9), again from ref. [57], we display the constraints on explicit $R$-parity-violating FCNCs for the opposite sign of $\delta m^{2}$. Though this does not represent as solid a constraint as that which follows from the SN1987A rate argument, it is clear that it would be much more stringent than any constraint obtained from the laboratory. In particular, putting together the results from Fig. (8) with those of Fig. (9), corresponding to two sign possibilities for $\delta m^{2}$, it is clear that they disfavour a leptoquark interpretation of the recent HERA anomaly.

Another example of how astrophysics can constrain neutrino properties has to do with shock reheating and the delayed explosion mechanism of Bethe and Wilson. This has been recently considered within the scenario of resonant $\nu_{e} \rightarrow \nu_{s}$ and $\bar{\nu}_{e} \rightarrow \bar{\nu}_{s}$ conversions in supernovae, where $\nu_{s}$ is a sterile neutrino [58], with mass in the hot dark matter mass range. In Fig. (10), taken from ref. [58], the 


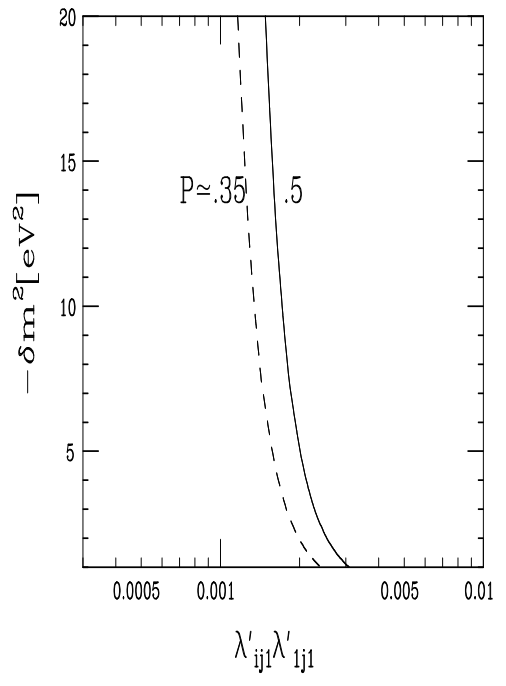

Figure 8: SN1987A bound on R-parity-violating neutrino interactions.

resulting constraints on mixing and mass difference for the $\nu_{e}-\nu_{s}$ system that follow from the supernova shock re-heating argument are displayed. The implications of such a scenario for the detected $\bar{\nu}_{e}$ signal from SN1987A and for the $r$-process nucleosynthesis hypothesis have also been analysed in ref. [58]. An interesting result is that SN $r$-process nucleosynthesis can be enhanced for some region of active-sterile oscillation parameters. As a final remark I mention a nice recent paper [59] showing how resonant neutrino conversions [60] induced by Majorana neutrino transition moments [61] can also be restricted by SN arguments.

In summary, neutrino masses now seem required in order to account for the data on solar and atmospheric neutrinos, as well as the hot dark matter component of the Universe. The scenarios needed in order to reconcile these anomalies may have interesting implications [62]. Detecting neutrino masses is one of the most outstanding goals in particle physics, with far-reaching implications also for the understanding of fundamental issues in astrophysics and cosmology. This work was supported by DGICYT grant PB95-1077 and by EEC under the TMR contract ERBFMRX-CT96-0090.

\section{References}

[1] R.N. Mohapatra and G. Senjanovic, Phys. Rev. D23 (1981) 165 and references therein.

[2] J.C. Pati, A. Salam. Phys. Rev. D8 (1973) 1240

[3] M Gell-Mann, P Ramond, R. Slansky, in Supergravity, ed. D. Freedman et al. (1979); T. Yanagida, in KEK lectures, ed. O. Sawada et al. (1979)

[4] J. Schechter and J. W. F. Valle, Phys. Rev. D22 (1980) 2227 


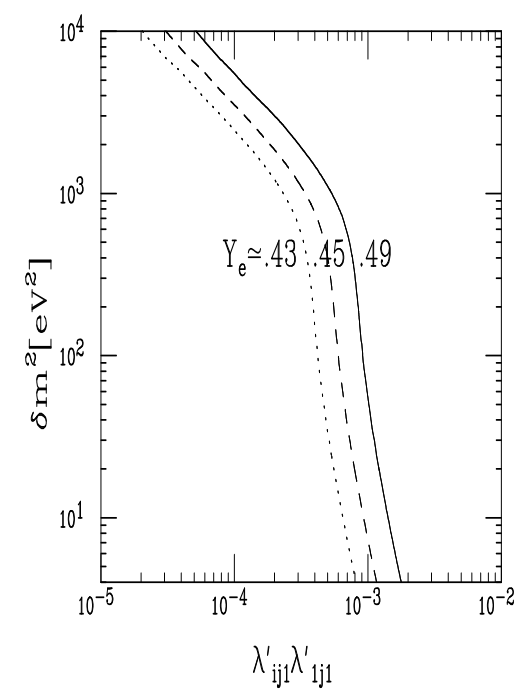

Figure 9: Supernova r-process restriction on $\mathrm{R}$-parity-violating neutrino interactions.

[5] A. Ioannissyan, J.W.F. Valle, Phys. Lett. B332 (1994) 93-99; B. Bamert, C.P. Burgess, Phys. Lett. B329 (1994) 289; D. Caldwell and R. N. Mohapatra, Phys. Rev. D50 (1994) 3477; D. G. Lee and R. N. Mohapatra, Phys. Lett. B329 (1994) 463; A. S. Joshipura, Phys. Rev. D51 (1995) 1321

[6] D. Wyler, L. Wolfenstein, Nucl. Phys. B218 (1983) 205

[7] R. Mohapatra, J. W. F. Valle, Phys. Rev. D34 (1986) 1642; J. W. F. Valle, Nucl. Phys. B (Proc. Suppl.) B11 (1989) 118-177

[8] J.W.F. Valle, Phys. Lett. B199 (1987) 432

[9] H. Nunokawa, Y.Z. Qian, A. Rossi, J.W.F. Valle, Phys. Rev. D54 (1996) 4356-4363

[10] M. C. Gonzalez-Garcia, J. W. F. Valle, Phys. Lett. B216 (1989) 360.

[11] J. Bernabeu, A. Santamaria, J. Vidal, A. Mendez, J. W. F. Valle, Phys. Lett. B187 (1987) 303; J. G. Korner, A. Pilaftsis, K. Schilcher, Phys. Lett. B300 (1993) 381

[12] G. C. Branco, M. N. Rebelo, J. W. F. Valle, Phys. Lett. B225 (1989) 385; N. Rius, J. W. F. Valle, Phys. Lett. B246 (1990) 249

[13] M. Dittmar, M. C. Gonzalez-Garcia, A. Santamaria, J. W. F. Valle, Nucl. Phys. B332 (1990) 1; M. C. Gonzalez-Garcia, A. Santamaria, J. W. F. Valle, ibid. B342 (1990) 108; J. Gluza, M. Zralek; Phys. Rev. D55 (1997) 7030-7037

[14] M. C. Gonzalez-Garcia, J. W. F. Valle, Mod. Phys. Lett. A7 (1992) 477; erratum Mod. Phys. Lett. A9 (1994) 2569; A. Ilakovac, A. Pilaftsis, Nucl. Phys. B437 (1995) 491; A. Pilaftsis, Mod. Phys. Lett. A9 (1994) 3595

[15] L. Hall, V. Kostelecky, S. Raby, Nucl. Phys. B267 (1986) 415

[16] A. Zee, Phys. Lett. B93 (1980) 389; K. S. Babu, Phys. Lett. B203 (1988) 132

[17] J. T. Peltoniemi, and J W F Valle, Phys. Lett. B304 (1993) 147; 


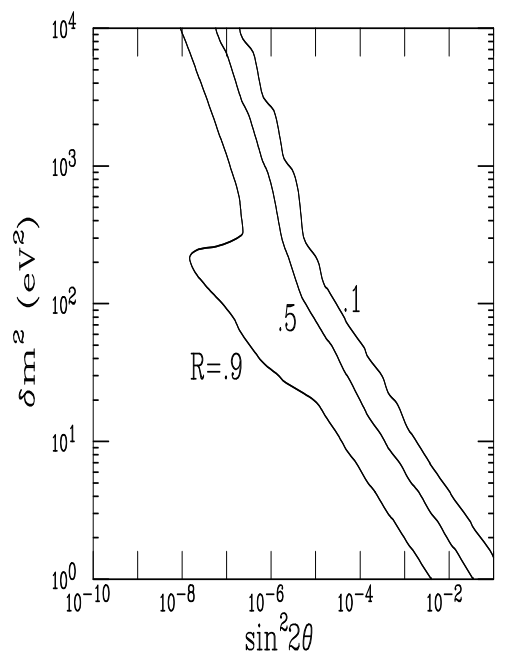

Figure 10: Supernovae and sterile neutrinos.

[18] M. A. Díaz, J. C. Romão and J. W. F. Valle, hep-ph/9706315; K. S. Babu, A. Joshipura, in preparation

[19] F. Vissani and A. Yu. Smirnov, Nucl. Phys. B460 (1996) 37-56; H.P. Nilles and N. Polonsky, Nucl. Phys. B484 (1997) 33; B. de Carlos, P.L. White, Phys. Rev. D55 (1997) 4222-4239; T. Banks, T. Grossman, E. Nardi, Y. Nir, Phys. Rev. D52 (1995) 5319; E. Nardi, Phys. Rev. D55 (1997) 5772; S. Roy and B. Mukhopadhyaya, Phys. Rev. D55 (1997) 7020.

[20] J. C. Romão, J. W. F. Valle, Nucl. Phys. B381 (1992) 87-108

[21] M. A. Diaz, A. Joshipura, J. W. F. Valle, in preparation

[22] A. Santamaria, J.W.F. Valle Phys. Lett. 195B (1987) 423; Phys. Rev. Lett. 60 (1988) 397

[23] R. Hempfling, Nucl. Phys. B478 (1996) 3; K. S. Babu, A. Joshipura, in preparation

[24] A. Joshipura and J. W. F. Valle, Nucl. Phys. B397 (1993) 105 and references therein.

[25] Particle Data Group, Phys. Rev. D54 (1996) 1-720 (see also URL: http://pdg.lbl.gov/).

[26] V. M. Lobashev, these proceedings

[27] K. Assamagan et al, Phys. Rev. D53 (1996) 6065

[28] I. Nikolic, talk at WIN97, Capri and references therein.

[29] J. Gómez-Cadenas, M. C. González-García, Phys. Rev. D39 (1989) 1370; J. Gómez-Cadenas et al., Phys. Rev. D41 (1990) 2179

[30] B. Achkar et al Nucl. Phys. B434 (1995) 503; G.S. Vidyakin et al JETP 59 (1994) 390.

[31] Karmen collaboration, B. Armbruster et al. Nucl. Phys. B (Proc. Suppl.) B38 (1995) 235.

[32] C. Athanassopoulos, Phys. Rev. Lett. 75 (1995) 2650; J. E. Hill, Phys. Rev. Lett. 75 (1995) 2654;

[33] N. Ushida et al, Phys. Rev. Lett. 57 (1986) 2897; L. Borodovsky et al, Phys. Rev. Lett. 68 (1992) 274. 
[34] LSND collaboration, nucl-ex/9706006; W. Louis, these proceedings

[35] H. Klapdor, these proceedings

[36] W. Haxton, Nucl. Phys. B (Proc. Suppl.) 31 (1993) 88.

[37] L. Wolfenstein, Nucl. Phys. B186 (1981) 147; J. W. F. Valle, Phys. Rev. D27 (1983) 1672 and references therein

[38] J. Schechter and J. W. F. Valle, Phys. Rev. D25 (1982) 2951

[39] M. Hirsch, H.V. Klapdor-Kleingrothaus, S.G. Kovalenko, hep-ph/9707207.

[40] E. Kolb, M. Turner, The Early Universe, Addison-Wesley, 1990, and references therein

[41] J. Bardeen, J. Bond and G. Efstathiou, Astrophys. J. 321 (1987) 28; J. Bond and G. Efstathiou, Phys. Lett. B265 (1991) 245; M. Davis et al., Nature 356 (1992) 489; S. Dodelson, G. Gyuk and M. Turner, Phys. Rev. Lett. 72 (1994) 3754; H. Kikuchi and E. Ma, Phys. Rev. D51 (1995) 296; H. B. Kim and J. E. Kim, Nucl. Phys. B433 (1995) 421; M. White, G. Gelmini and J. Silk, Phys. Rev. D51 (1995) 2669; A. S. Joshipura and J. W. F. Valle, Nucl. Phys. B440 (1995) 647.

[42] Y. Chikashige, R. Mohapatra, R. Peccei, Phys. Rev. Lett. 45 (1980) 1926

[43] For a review see, for example, J. W. F. Valle, Gauge Theories and the Physics of Neutrino Mass, Prog. Part. Nucl. Phys. 26 (1991) 91-171

[44] J. Schechter, J. W. F. Valle, Phys. Rev. D25 (1982) 774

[45] J. W. F. Valle, Phys. Lett. B131 (1983) 87; G. Gelmini, J. W. F. Valle, Phys. Lett. B142 (1984) 181; J. W. F. Valle, Phys. Lett. B159 (1985) 49; M. C. Gonzalez-Garcia, J. W. F. Valle, Phys. Lett. B216 (1989) 360; A. Joshipura, S. Rindani, Phys. Rev. D46 (1992) 3000

[46] R.F. Carswell, M. Rauch, R.J. Weynman et al., MNRAS 268 (1994) L1; A. Songalia, L.L. Cowie, C. Hogan and M. Rugers, Nature 368 (1994) 599; D. Tytler and X.M. Fan, Bull. Am. Astr. Soc. 26 (1994) 1424; D. Tytler, talk at the Texas Symposium, December 1996.

[47] N. Hata et al., Phys. Rev. Lett. 75 (1995) 3977; C.J. Copi, D.N. Schramm and M.S. Turner, Science 267 (1995) 192 and Phys. Rev. Lett. 75 (1995) 3981; K. A. Olive and G. Steigman, Phys. Lett. $B 354$ (1995) 357-362; S. Sarkar, Rep. on Prog. in Phys. 59 (1997) 1493; P. J. Kernan and S. Sarkar, Phys. Rev. D 54 (1996) R3681

[48] E. W. Kolb, M. S. Turner, A. Chakravorty and D. N. Schramm, Phys. Rev. Lett. 67 (1991) 533; A.D. Dolgov and I.Z. Rothstein, Phys. Rev. Lett. 71 (1993) 476.

[49] A.D. Dolgov, S. Pastor, and J.W.F. Valle, Phys. Lett. B383 (1996) 193-198; hep-ph/9602233

[50] A.D. Dolgov, S. Pastor, J.C. Romão and J.W.F. Valle, Nucl. Phys. B496 (1997) 24-40

[51] A Masiero, J. W. F. Valle, Phys. Lett. B251 (1990) 273; J. C. Romao, C. A. Santos, and J. W. F. Valle, Phys. Lett. B288 (1992) 311;

[52] For a recent ref. see S. Hannestad, hep-ph/9711249; for a review see G. Steigman; in Cosmological Dark Matter, p. 55, World Scientific, 1994, ISBN 981-02-1879-6

[53] A. B. Balantekin, Selected Topics in Neutrino Astrophysics astro-ph/9706256

[54] G. Raffelt, Stars as laboratory for fundamental physics, Univ. of Chicago Press, (1996). 
[55] Y.-Z. Qian, G. M. Fuller, G. J. Mathwes, R. W. Mayle, J. R. Wilson and S. E. Woosley, Phys. Rev. Lett. 71 (1993) 1965-1968

[56] A. Yu. Smirnov, D. N. Spergel and J. N. Bahcall, Phys. Rev. D49 (1994) 1389.

[57] H. Nunokawa, A. Rossi and J. W. F. Valle, Nucl. Phys. B482 (1996) 481.

[58] H. Nunokawa, J.T. Peltoniemi, A. Rossi, J.W.F. Valle, Phys. Rev. D56 (1997) 1704-1713

[59] H. Nunokawa, Y.Z. Qian, G.M. Fuller, Phys. Rev. D55 (1997) 3265-3275

[60] C.S. Lim and W.J. Marciano, Phys. Rev. D37 (1988) 1368; E.Kh. Akhmedov, JETP 68 (1989) 690 .

[61] J. Schechter and J.W.F. Valle, Phys. Rev. D24 (1981) 1883; Phys. Rev. D25 (1982) 283.

[62] J. W. F. Valle, e-Print Archive: hep-ph/9709365 